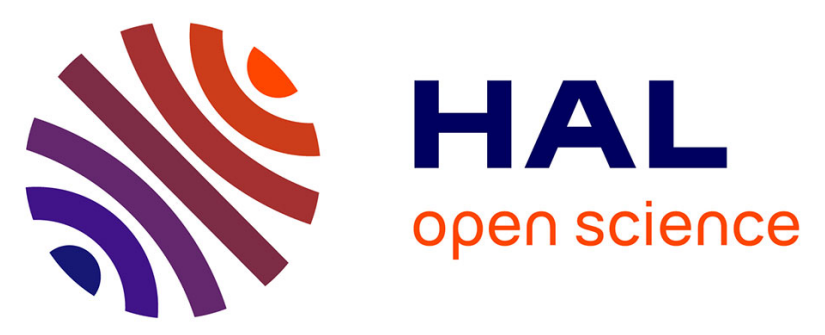

\title{
Automatic Alignment of pre and intraoperative Data using Anatomical Landmarks for Augmented Laparoscopic Liver Surgery
}

Rosalie Plantefeve, Nazim Haouchine, Jean Pierre Radoux, Stéphane Cotin

\section{- To cite this version:}

Rosalie Plantefeve, Nazim Haouchine, Jean Pierre Radoux, Stéphane Cotin. Automatic Alignment of pre and intraoperative Data using Anatomical Landmarks for Augmented Laparoscopic Liver Surgery. International Symposium on Biomedical Simulation ISBMS, Oct 2014, Strasbourg, France. hal01068246

\section{HAL Id: hal-01068246 \\ https://hal.inria.fr/hal-01068246}

Submitted on 25 Sep 2014

HAL is a multi-disciplinary open access archive for the deposit and dissemination of scientific research documents, whether they are published or not. The documents may come from teaching and research institutions in France or abroad, or from public or private research centers.
L'archive ouverte pluridisciplinaire $\mathbf{H A L}$, est destinée au dépôt et à la diffusion de documents scientifiques de niveau recherche, publiés ou non, émanant des établissements d'enseignement et de recherche français ou étrangers, des laboratoires publics ou privés. 


\title{
Automatic Alignment of pre and intraoperative Data using Anatomical Landmarks for Augmented Laparoscopic Liver Surgery
}

\author{
Rosalie Plantefève ${ }^{12}$, Nazim Haouchine ${ }^{23}$, Jean-Pierre Radoux ${ }^{1}$, and \\ Stephane Cotin 234 \\ 1 MEDIC@, ALTRAN, France \\ 2 Shacra Team, INRIA, France \\ 3 Lille University, France \\ 4 IHU Strasbourg, France
}

\begin{abstract}
Each year in Europe 50,000 new liver cancer cases are diagnosed for which hepatic surgery combined to chemotherapy is the most common treatment. In particular the number of laparoscopic liver surgeries has increased significantly over the past years. This type of minimally invasive procedure which presents many benefits for the patient is challenging for the surgeons due to the limited field of view. Recently new augmented reality techniques which merge preoperative data and intraoperative images and permit to visualize internal structures have been proposed to help surgeons during this type of surgery. One of the difficulties is to align preoperative data with the intraoperative images. We propose in this paper a semi-automatic approach for solving the ill-posed problem of initial alignment for Augmented Reality systems during liver surgery. Our registration method relies on anatomical landmarks extracted from both the laparoscopic images and three-dimensional model, using an image-based soft-tissue reconstruction technique and an atlas-based approach, respectively. The registration evolves automatically from a quasi-rigid to a non-rigid registration. Furthermore, the surface-driven deformation is induced in the volume via a patient specific biomechanical model. The experiments conducted on both synthetic and in vivo data show promising results with a registration error of $2 \mathrm{~mm}$ when dealing with a visible surface of $30 \%$ of the whole liver.
\end{abstract}

\section{Introduction}

Context: Minimally invasive surgery is regarded as one of the major advances in surgery of last decades considering the benefits for patient in term of time recovery and reduced pain. Yet, one problem remains: the surgeon still needs to register mentally the preoperative data onto the laparoscopic view to determine the tumours and vessels location. The introduction of optics in the clinical routines have motivated several research groups to investigate the benefits of Augmented Reality to help surgeons during the procedure [1]. Indeed, Augmented Reality has the potential to provide an enriched visual feedback to the surgeon by the fusion of intraoperative images and three-dimensional preoperative data such as tumours and vascular network. 
Previous work: In this context several methods have been proposed to solve the numerous challenges that present the abdominal cavity, ranging from occluded regions due to instrument [2], organ tracking disturbance due to blur or smoke [3], and unpredictable deformations produced by heart beating, breathing or manipulation with instruments [4]. However, despite these numerous techniques, very few works have investigated the initial alignment between the laparoscopic images and the three-dimensional model. Indeed, the initialisation is often assumed to be done manually $[2,4,5]$, which can be a major source of error given the significant deformations that the organs may undergo during the surgery. In addition, since gas is insufflated (pneumoperitoneum) to increase the working space [6], the preoperative data may no longer correspond to the intraoperative image. In order to optimize the trocars placement on the skin, in [6] it is proposed to simulate the pneumoperitoneum using only a preoperative model and patient-specific biomechanical parameters. The evaluation of their method on real data highlights its potential usability for Augmented Reality. Based on this work, Oktay et al. [7] propose to exploit the intraoperative data acquired from CT-scans after insufflation as an additional constraint to drive the simulation. Although this method provides accurate registration, it relies on intraoperative scans. Another intraoperative registration method is presented in [8]. The authors use an intraoperative ultrasound probe to register the vessel tree on a three dimentional liver model. However, since our aim is to register the laparoscopic data recorded by a camera, such information is not available. Clements et al. [9] introduced an ICP-based approach to aid in the initial pose estimation. Using salient anatomical features, identifiable in both the preoperative images and intraoperative liver surface data, this method is able to reach a reasonable solution, but is restrained to rigid transformations. To handle deformations, biomechanical models are often used to regularize the registration methods. For instance, in [10] a registration of intraoperative MR brain images is proposed where the model is based on linear elasticity and the finite element method. Moreover the method proposed by Clements relies on manual estimation of anatomical structures position which is too cumbersome to be done in a clinical context. A solution could be to use anatomical atlases to gather information about features positions. In [11] the authors use a histological atlas containing detailed anatomical information to target small brain structure in a pre operative MRI for neurosurgery planning.

\section{Methods}

The overall computational flow of our method involves two main steps: detection of anatomical landmarks on both laparoscopic images and preoperative data, and a nonrigid feature-based registration similar to the Iterative Closest Point (ICP).

\subsection{Pre and Intraoperative Data Computation}

We define a set of landmarks that are chosen to be visible on stereoscopic images and in at least one preoperative imaging technique (see Fig. 1). These landmarks will be labelled on one frame of the endoscopic data and on the three-dimensional model. 


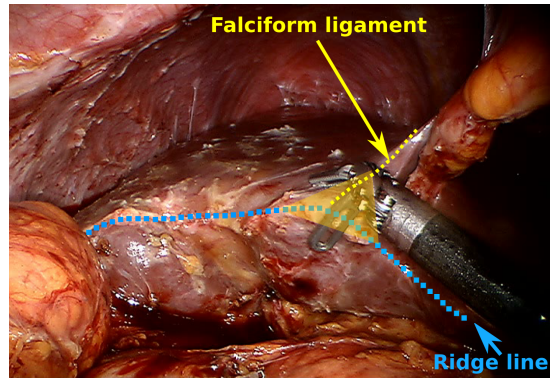

(a) Laparoscopic view

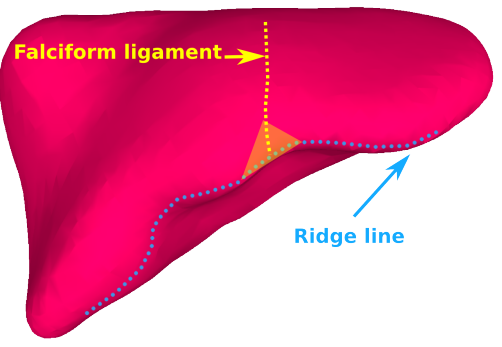

(b) three-dimensional model

Fig. 1. Our registration method must acurately register the three-dimensional model onto the laparoscopic view. The ridge line and a section of the falciform ligament are used as anatomical features to guide the registration process.

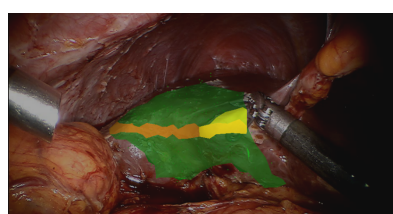

(a)

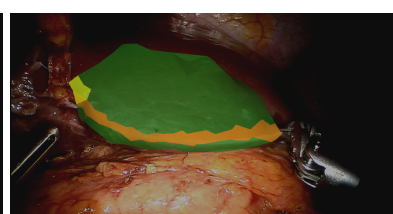

(b)

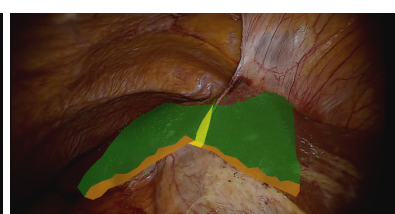

(c)

Fig. 2. Detection of anatomical landmarks on laparoscopic images: The liver surface (in green), the ridge line (in orange) and the falciform ligament (in yellow) are extracted from the laproscopic image using stereo matching method to obtain a 3D labelled point cloud.

Stereo Endoscopic Scene Estimation: On one of the stereo endoscopic images, the falciform ligament, the ridge line and the liver surface are defined manually as anatomical landmarks of the liver. The operator selects (e.g. by clicking) some points on the border of the regions of interest to form closed areas. This selection takes only a few seconds. Once the targeted regions are selected, we build for each of them a three-dimensional point cloud using the method described in [4] that relies on a sparse stereo matching technique to recover the liver surface shape. These point clouds are labelled according to their corresponding regions as illustrated in Fig. 2.

Preoperative Anatomical Landmarks Detection: The three-dimensional model is obtained form the CT images via a standard semi-automatic segmentation process described in [12]. The resulting mesh is then augmented with the same anatomical landmarks as the ones detected in the stereoscopic images. For the liver one landmark is automatically detected on the three-dimensional model: the ridge line. This landmark can be easily identified in most human livers. The automatic detection of the ridge line on the three-dimensional model of the liver is performed as follows: the edges separating two triangles with sufficiently different normals are selected as seeds. The definition of the threshold is based on statistics on the normals differences for the whole mesh. 
Then, the ridge line is extended from the seed edges; if no extension is found the seeds are removed. Iteratively the ridge line is reconstructed. The other landmarks (only the falciform ligament for the liver) are transferred via a statistical atlas of the liver surface containing the landmarks location [13]. This atlas is constructed with a set of pre operative medical images of the organ of interest. The imaging modality should be chosen such that the selected anatomical landmarks are visible. For each image an expert perform a manual segmentation of the organ and anatomical landmarks. Then all the segmentations are aligned in a common reference frame and the mean shape and standard deviations are computed. This atlas is finally registered on the three-dimensional model.

\subsection{Feature-based Registration}

Our approach relies on a non-rigid registration which computes the elastic transformation that maximizes the shape similarity between the source and target configurations. One ICP is computed per labelled area and they are all solved simultaneously.

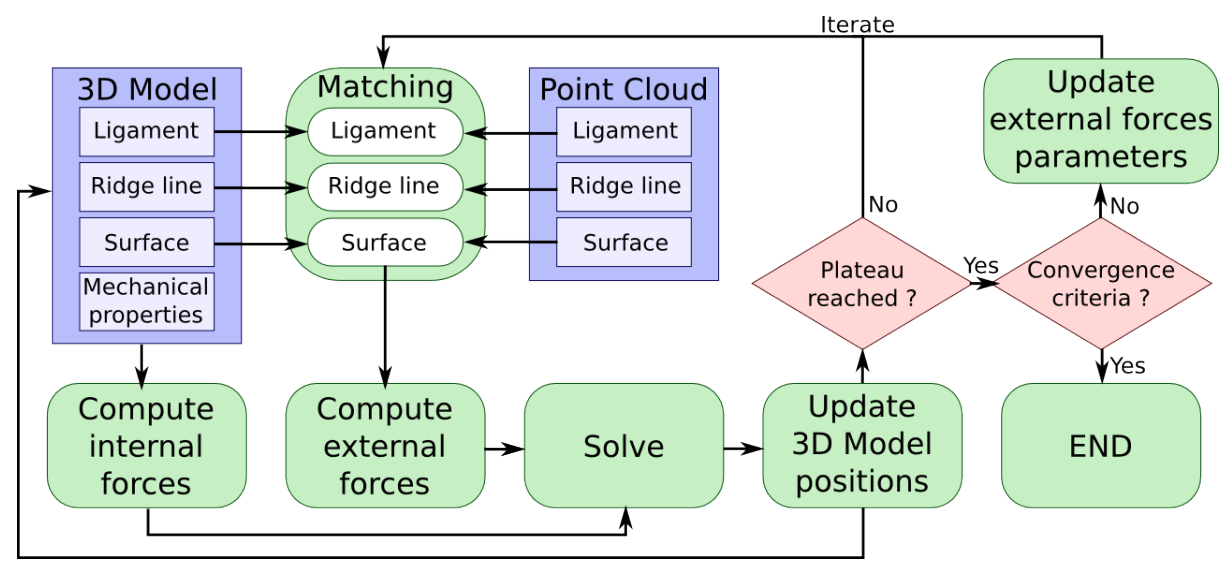

Fig. 3. Main steps of the ICP-like registration method.

Matching: The target model is the sets of points extracted from the stereoscopic images and the source model is the organ three-dimensional model. Three matchings are achieved simultaneously: one for each labelled area. For each area the points of the target model are projected on their corresponding area on the source model surface as shown in Fig. 3. We do not project the source model points on the target since the target only corresponds to a part of the source model. The target points are projected onto the triangles of the source model surface. A matching pair $\left\{p_{t}, p_{s}\right\}$ consisting of the target point and its closest projection is then defined. The outliers are pruned with relative distance and normal thresholds. The relative distance thresholds keep the pairs points for which $\left\|\overrightarrow{p_{t} p_{s}}\right\|<d_{t} \max \left(\left\{\left\|\overrightarrow{p_{t} p_{s}}\right\|\right\}\right.$ allpairs $), d_{t} \in[0 ; 1]$ where $\|\cdot\|$ is the Euclidean norm. 
The normal thresholds prune the pairs for which their normal dot product $\widehat{n}_{p_{t}} \cdot \widehat{n}_{p_{s}}<n_{t}$, $n_{t} \in[0 ; 1]$. These thresholds must be set according to the deformation characteristics and they should be smaller for larger deformations. The ICP algorithm aims at reducing the global distance between the $\left\{p_{t}, p_{s}\right\}$ pairs.

Model of deformation: A biomechanical model is associated to the augmented threedimensional model. As the three-dimensional model and the points extracted from the stereoscopic images are two representations of the same liver, they share the same biomechanical behaviour. Different constitutive laws can be used to model the liver such as hyperelastic formulation [14]. For computational efficiency we rely on a corotational formulation as proposed by [15]. A fast finite element approach is used to compute the deformation of the organ. This computation can be performed very efficiently thus making it very suitable for the ICP algorithm. Regarding the parameters of the model, the Young's modulus can be patient specific (e.g. obtained by elastography) or is set to the average value found in the literature [16]. The Poisson ratio is set to 0.4 as the liver is nearly incompressible (fluid exchanges allow for some volume variation).

Definition of constraints: The constraints are imposed on the system with a penalty force:

$$
\mathbf{f}=\sum_{i}^{m} \mathbf{f}_{i}, \quad \text { (1) } \quad \text { with } \quad \mathbf{f}_{i}=\sum_{j}^{p_{i}} f_{i} \arctan \left(k \mathbf{x}_{i j}\right)
$$

where $m$ is the number of labelled area, $p_{i}$ the number of corresponding pairs in the area $i, f_{i}$ a penalty factor, $k$ a scale factor and $\mathbf{x}_{i j}$ the vector between the $\mathrm{j}$-th target point and its projection. The $k$ value is related to the point cloud noise and is used to avoid overfitting as a lower value decrease the force intensity for small distances. When registering non-rigidly the complete surface model w.r.t. the reconstructed part, the process may suffer from inaccuracy due to the limited knowledge of correspondence between the two. Therefore, the labelled areas are penalized differently to use the anatomical landmarks as anchor points for the registration. Moreover, all the $f_{i}$ are not constant over time to ensure that the registration evolves from a quasi-rigid (i.e. the applied forces are too small to produce a significant deformation) to non-rigid state. During the registration we detect when the mechanical system has reached a plateau and then update the $f_{i}$ values. In case of liver, the falciform ligament is the most reliable anatomical feature. It also corresponds to a very small area and therefore does not contribute much to the deformation. For that reason, $f_{i}$ corresponding to this landmark is constant during the registration process. The other $f_{i}$ are of the form $\frac{2 f_{i_{\max }}\left(\frac{n}{v_{i}}\right)^{2}}{\left(\frac{n}{v_{i}}\right)^{2}+1}$ for $n<v_{i}$ (i.e. they are set to 0 at the beginning of the registration) and $f_{i_{\max }}$ for $n>v_{i}$ where $n$ is the plateau index and $v_{i}$ a speed coefficient. $v_{i}$ and $f_{i_{\max }}$ must be set so that the objective function is more convex in the early stage of registration and that the minimum become more precise over time. Therefore, the $v_{i}$ and $f_{i_{\max }}$ of small area must be smaller than those corresponding to the large area. According to our tests, the algorithm shows low sensitivity w.r.t. the parameters. The registration is stopped when $n=\max \left(v_{i}\right)+1$.

Registration method: At each simulation step the following equation is solved

$$
\mathbf{K}(\mathbf{u}) \mathbf{u}=\mathbf{f}
$$


where $\mathbf{u}$ is the displacement vector, $\mathbf{K}$ the non-linear stiffness provided by the FE formulation and $\mathbf{f}=\frac{d E(\mathbf{u})}{d \mathbf{u}}$ is the vector of constraint forces. The pairing is then recomputed generating a new force. Finally, during the simulation the anatomical landmarks provide a coarse registration that improves the robustness of the ICP method, whereas the biomechanical model plays a role of regularization and allows for an accurate solution of local deformations.

\section{Results}

This section presents the results obtained using our method. We conduct experiments on two different type of data: computer-generated data and liver data during in vivo surgical procedure. For convergence an average of 3700 iterations is needed. Each iteration takes $700 \mathrm{~ms}$ on average and the solver represent around $20 \%$ of a time step. In order to insure the success of the method, the anatomical features must be at least partially visible.

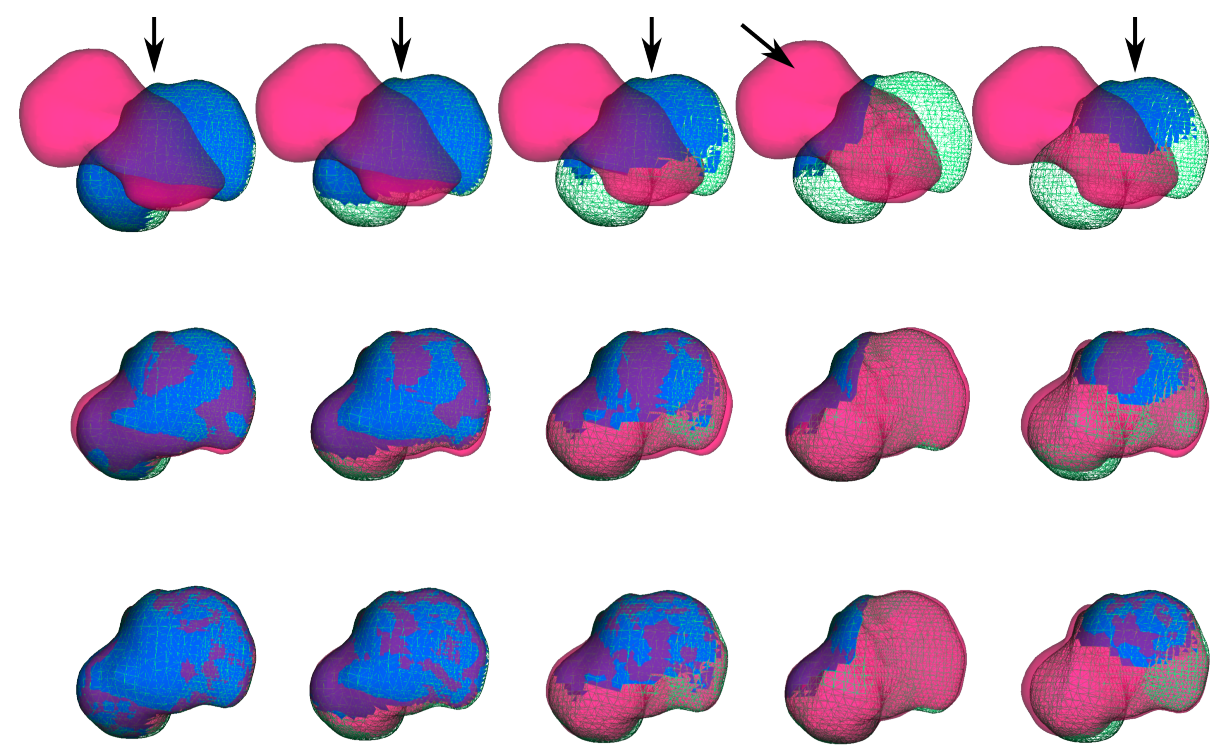

Fig. 4. Registration results on synthetic data. The source model is in transparent red, the target surface used for the registration is in blue and the whole target surface in green wireframe. The first row shows the initial pose, the second row the result after the quasi-rigid registration and the last row the final result. Each column shows the results for different target surface areas. The target surface of the columns from left to right correspond to $50 \%, 40 \%, 30 \%, 20 \%$ and $10 \%$ of the whole surface. The black arrows indicate the virtual camera angle. 


\subsection{Evaluation with in silico data}

We evaluated our method by registering a three-dimensional model of the liver segmented from CT-scans (source) on the same model that had undergone both a rigid and non-rigid transformations (target). In order to obtain the target liver, we apply a pressure on the surface of the model to simulate two different deformations possibly induced by the pneumoperitoneum, and a random rigid transformation (cf Fig. 4). In real situations only partial surface information is acquired by the laparoscopic camera. To evaluate the amount of information needed to achieve an accurate registration faces of the deformed model are deleted to keep only a portion of its surface from $50 \%$ to $10 \%$.

The results presented in Fig. 5 show that even with only 10\% of visible surface and a poor initial alignment our method is able to perform the registration with a mean error lower than $4 \mathrm{~mm}$. The deformation 1 was more important on the liver face opposed to the camera view. This fact could explain the lower quality of the results. The plot also suggests that for a small deformation the non-rigid part of the method does not introduce any improvement when compared to the quasi-rigid part as soon as $20 \%$ or less of the surface is visible. In these two cases the visible area underwent a small deformation comparatively to the other cases. However, the mean Hausdorff distance calculated between the registered three-dimensional model and the visible part does not exceed $0.3 \mathrm{~mm}$.

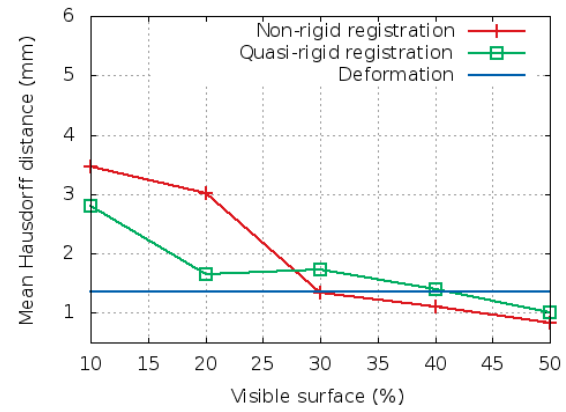

(a) Deformation 1

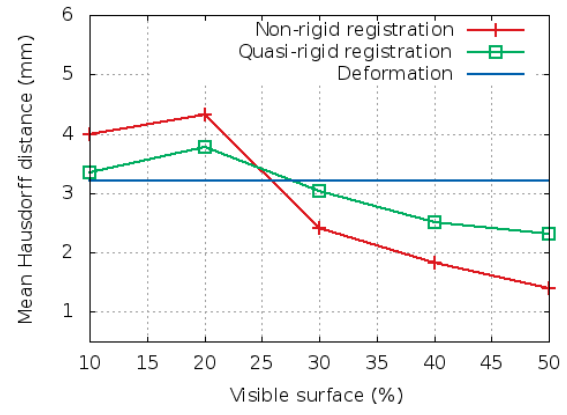

(b) Deformation 2

Fig. 5. Registration results on synthetic data showing the error w.r.t. the variation in visible surface

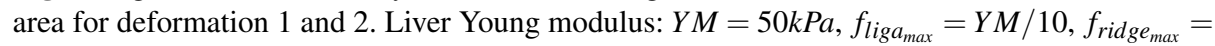
$Y M / 3, v_{\text {ridge }}=2, f_{\text {surf }_{\max }}=2 Y M, v_{\text {surf }}=25, d_{t}=0.95, n_{t}=0$ and $k=2 \pi .10^{3}$ (90\% of the force intensity at $10^{-3} \mathrm{~mm}$ ). The blue lines represent the mean Hausdorff distance between the non deformed source model and the same model after deformation.

\subsection{Experiments with in vivo data}

To assess our approach in a real surgical environment (specular lights, instrument occlusions ...) and to evaluate the ability of our non-rigid registration to estimate the 
initial pose, we tested our method on in vivo laparoscopic images of a human liver. The results illustrated in Fig. 6 report a visually correct initial non-rigid registration of the liver model on the laparoscopic image with only a partial and noisy three-dimensional surface reconstruction. For this simulation we used the same parameter values as for the in silico data (see the caption of Fig. 5) except for $k=2 \pi \cdot 10^{1}(90 \%$ of the force intensity at $10^{-1} \mathrm{~mm}$ ). The mean hausdorff distance between the point clouds and the three-dimensional model is $0.5 \mathrm{~mm}$ and $0.6 \mathrm{~mm}$ for the cases (a) and (b) of Fig. 6 respectively.

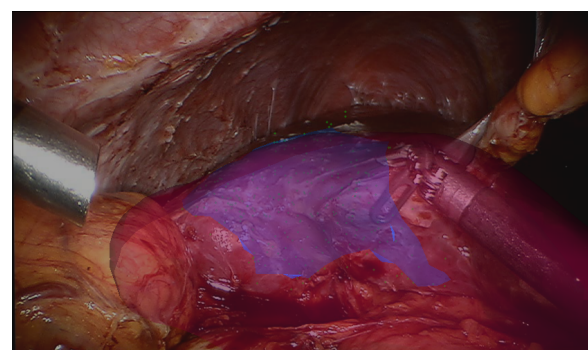

(a)

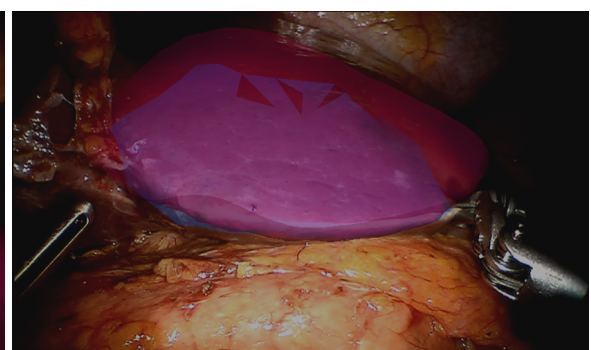

(b)

Fig. 6. Registration results on in vivo data on two different views of a human liver. The registered mesh is shown in red while the partial reconstructed patch is depicted in blue.

\section{Conclusion}

In this paper, we proposed a semi-automatic method for initial alignment of an organ preoperative three-dimensional model onto an endoscopic view of the same organ. This method relies on a feature-based registration framework which evolves from a quasirigid state to a non rigid state. The anatomical features used to guide the registration are selected manually on the endoscopic view and are transferred via an atlas to the three-dimensional model. We evaluated the method using ten synthetic data and two real endoscopic views. The results show that the method deforms accurately the threedimensional model when the visible surface corresponds to $30 \%$ or more of the entire organ surface. It was also demonstrated that the registration works with real endoscopic data. In the future, we will add information about the organ silhouette. We also plan to limit the constraint forces applied during the registration according to real forces imposed on the organ boundary during the intervention.

\section{Acknowledgement}

The authors would like to thank the anonymous reviewers for their valuable comments and suggestions and Igor Peterlik for his thorough re-reading of this article. 


\section{References}

1. Nicolau, S., Soler, L., Mutter, D., Marescaux, J.: Augmented reality in laparoscopic surgical oncology. Surgical Oncology 20(3) (2011) 189 - 201

2. Puerto-Souza, G., Mariottini, G.: Toward long-term and accurate augmented-reality display for minimally-invasive surgery. In: ICRA 2013. (2013) 5384-5389

3. Yip, M.C., Lowe, D.G., Salcudean, S.E., Rohling, R., Nguan, C.Y.: Tissue tracking and registration for image-guided surgery. IEEE Trans. Med. Imaging 31(11) (2012) 2169-2182

4. Haouchine, N., et al.: Image-guided simulation of heterogeneous tissue deformation for augmented reality during hepatic surgery. In: ISMAR. (2013) 199-208

5. Su, L.M., et al.: Augmented reality during robot-assisted laparoscopic partial nephrectomy: Toward real-time 3d-ct to stereoscopic video registration. Urology 73(4) (2009) 896 - 900

6. Bano, J., et al.: Simulation of pneumoperitoneum for laparoscopic surgery planning. In: MICCAI 2012. (2012) 91-98

7. Oktay, O., et al.: Biomechanically driven registration of pre- to intra-operative $3 \mathrm{~d}$ images for laparoscopic surgery. In: MICCAI 2013. (2013)

8. Dagon, B., Baur, C., Bettschart, V.: A framework for intraoperative update of $3 \mathrm{~d}$ deformable models in liver surgery. In: Engineering in Medicine and Biology Society, 2008. EMBS 2008. 30th Annual International Conference of the IEEE, IEEE (2008) 3235-3238

9. Clements, L.W., et al.: Robust surface registration using salient anatomical features for image-guided liver surgery: Algorithm and validation. Medical Physics 35(6) (2008)

10. Ferrant, M., et al.: Registration of 3-d intraoperative mr images of the brain using a finiteelement biomechanical model. IEEE Trans. on Medical Imaging 20(12) (2001) 1384-1397

11. Bardinet, E., et al.: A three-dimensional histological atlas of the human basal ganglia. atlas deformation strategy and evaluation in deep brain stimulation for parkinson disease. J. of Neurosurgery 110(2) (2009)

12. Yushkevich, P.A., Piven, J., Cody Hazlett, H., Gimpel Smith, R., Ho, S., Gee, J.C., Gerig, G.: User-guided 3D active contour segmentation of anatomical structures: Significantly improved efficiency and reliability. Neuroimage 31(3) (2006) 1116-1128

13. Plantefeve, R., Peterlik, I., Courtecuisse, H., Trivisonne, R., Radoux, J.P., Cotin, S.: Atlasbased transfer of boundary conditions for biomechanical simulation. In: MICCAI. (2014)

14. Marchesseau, S., et al.: Multiplicative jacobian energy decomposition method for fast porous visco-hyperelastic soft tissue model. In: MICCAI. (2010)

15. Felippa, C., Haugen, B.: A unified formulation of small-strain corotational finite elements: I. theory. Comput Meth Appl Mech Eng 194(21) (2005)

16. Yeh, W.C., et al.: Elastic modulus measurements of human liver and correlation with pathology. Ultrasound in medicine \& biology (2002) 